\title{
Intravascular ultrasound imaging of the coronary arteries: an in vitro evaluation of measurement of area of the lumen and atheroma characterisation
}

\author{
Mark H Anderson, Iain A Simpson, Demosthenes Katritsis, Michael J Davies, \\ David E Ward
}

\begin{abstract}
Objective-To assess the accuracy of measurement of area of the lumen, and sensitivity, and specificity of detection of atheroma in coronary arteries in vitro with a commercially available $20 \mathrm{MHz}$ intravascular ultrasound system.

Setting-A teaching hospital department of cardiology with the support of the department of cardiovascular pathology.

Procedure-10 segments of coronary artery were removed from cadaver hearts. Intravascular ultrasound imaging was performed at fixed levels and the vessels were then sectioned and photographed before histological preparation. An independent blinded observer measured luminal area and assessed the presence of atheroma on the intravascular ultrasound images of 76 vessel sections (304 quadrants). The sensitivity and specificity of detection of atheroma was assessed in comparison with the histologically prepared sections. Luminal areas from intravascular ultrasound, photographs of cross sections of the vessels and histological sections were compared with the technique of limits of agreement.
\end{abstract}

Results-Overall $36 \%$ of the 304 quadrants studied histologically had identifiable atheroma. Intravascular ultrasound sensitivity for atheroma was 0.593 and the specificity was 0.839 . The positive predictive value was 0.674 , and the relative risk $3 \cdot 139$. Values for area of the vessel lumen were on average $9 \cdot 4 \mathrm{~mm}^{2}$ (confidence interval (CI) $8 \cdot 6$ $10.2 \mathrm{~mm}^{2}$ ) larger than those measured from photographs and 10.7 (CI 9.8$11.6 \mathrm{~mm}^{2}$ ) larger than those measured from the histological sections.

Conclusions-The intravascular ultrasound system assessed in this study significantly overestimated coronary vessel luminal area and had low sensitivity and specificity for detection of atheroma. Improvements in image resolution are required before this system can provide useful information on coronary artery size and morphology.

(Br Heart J 1992;48:276-81)
The concept of imaging the coronary arteries from within the lumen with ultrasound is not new but early attempts were limited by catheter technology. ${ }^{1}$ The development over the last 15 years of intravascular interventional procedures such as angioplasty and atherectomy has renewed interest in intravascular imaging. The realisation that the distribution and structure of the atheromatous plaque are important factors affecting the success of these strategies spurred interest in intravascular ultrasound and angioscopy. Imaging of the vessel after the intervention would enable assessment of the response of the atheroma to the treatment and identification of features associated with satisfactory long-term outcome. Although angioscopy can achieve imaging in front of the catheter tip it provides information on surface appearance only whereas intravascular ultrasound has the potential advantage of providing information on the deeper composition of the vessel wall including atheromatous plaques. This tantalising prospect is responsible for the current explosion of interest in intravascular ultrasound techniques.

Recently published studies have shown excellent correlation of vessel measurements made by ultrasound with those obtained at angiography ${ }^{2}$ and by histological studies in vitro. ${ }^{3}$ Also some studies have suggested that detailed information about the composition of the plaque may be obtained. ${ }^{4}$ The goal of our study was to see whether we could match the promising results suggested by these clinical studies with a commercially available ultrasound system, when applied in vitro to coronary artery specimens. Specifically we aimed to assess the accuracy of luminal area measurements made by the system and its ability to identify plaque morphology when compared with histological examination.

\section{Method}

SPECIMEN PREPARATION

Ten hearts with a short length of ascending aorta and the coronary sinuses intact were removed from cadavers at post mortem examination. A piece of plastic tubing was placed in the aorta and elastic bands ensured a snug waterproof connection between the tube and the aorta. Fixative solution (10\% formaldehyde) was perfused into the aorta at a pres- 
Figure 1 Coronary artery specimen sutured in Perspex carrier.

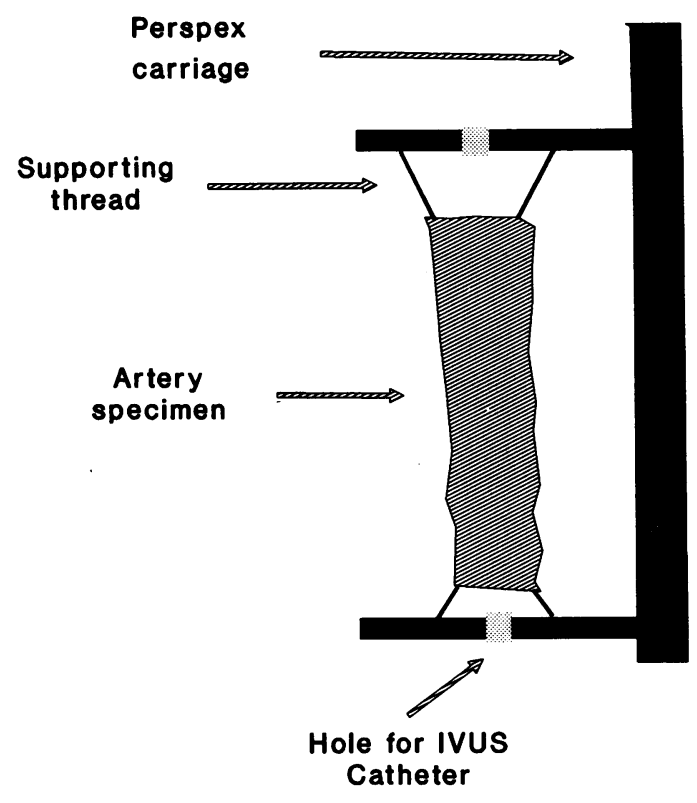

sure of $100 \mathrm{~mm} \mathrm{Hg}$ to simulate systemic pressure. The fixative drained into a lower reservoir from which it was recycled by a pump. After 24 hours connected to this system the heart was removed and a two $\mathrm{cm}$ long segment of coronary artery (nine hearts) or aortocoronary bypass graft (one heart) was removed for further study. This pressure fixation technique is used to maintain arterial morphology during subsequent processing.

Excess fat was dissected away from the segment of artery. The artery was suspended within the limbs of a Perspex carrier with $6^{\circ}$ prolene (fig 1). Sutures were adjusted so that the specimen was central but not under tension.

A Diasonics intravascular ultrasound system that provided the images ws connected to a $4 \cdot 8$ French gauge $20 \mathrm{MHz}$ ultrasound imaging catheter (Sonicath ${ }^{\mathrm{TM}}$, Boston Scientific Instruments). This catheter has a monorail tip to enable passage over a guide wire. Of necessity the ultrasound transducer was positioned some $3 \mathrm{~cm}$ back from the tip (fig 2). Before use the tip of this catheter was filled with sterile degassed water through a seal located within the monorail port in accordance with the manufacturer's

Figure $2 \quad x$ Ray appearance of the ultrasound imagin catheter tip. ( $A$ )

Ultrasound transducer; (B) fluid filled space; (C) radiopaque catheter tip;

(D) metal marker probe. instructions. The tip was then swung gently by hand to displace any small bubbles over the transducer. The proximal end of the catheter attaches to a motor drive unit which in turn is attached by flexible cable to the Diasonics ultrasound machine. The machine displays the image from the catheter on a six inch screen. Gain and magnification are variable. For both we used the settings recommended in the manufacturer's manual and these remained unchanged throughout the study. An on line facility allows measurement of circumference and area of outlines traced on the screen.

After preparation of the ultrasound imaging catheter the Perspex carriage supporting the artery specimen was placed in a small jig. The jig includes a water bath that was filled before addition of the carriage. A stainless steel screw mechanism enables the artery carriage to be raised or lowered precisely. The ultrasound imaging catheter was then placed into the water bath, passing through a small hole in the top of the artery carriage, down through the lumen of the artery, and through another small hole in the bottom of the carriage to finish with the tip just touching the bottom of the water bath (fig 3). The imaging catheter was supported so that it formed a smooth curve on its way to connect with the drive motor. For ease of manipulation the guide wire was omitted during this study.

\section{EXPERIMENTAL PROTOCOL}

The Perspex carriage supporting the artery specimen was slowly raised until an ultrasound image of the top of the specimen was just visible. Starting $2 \mathrm{~mm}$ from the top of each piece of artery, ultrasound images were taken at $3 \mathrm{~mm}$ intervals. The images were recorded on video tape and the luminal area was traced

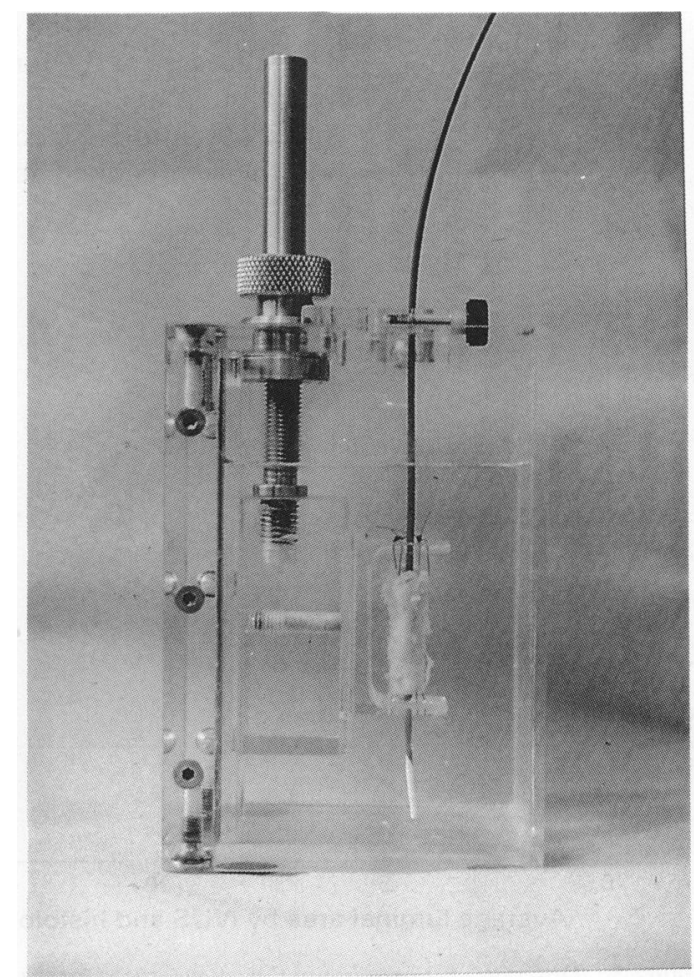

Figure 3 Jig with specimen carriage in place and ultrasound imaging catheter passing through specimen. 


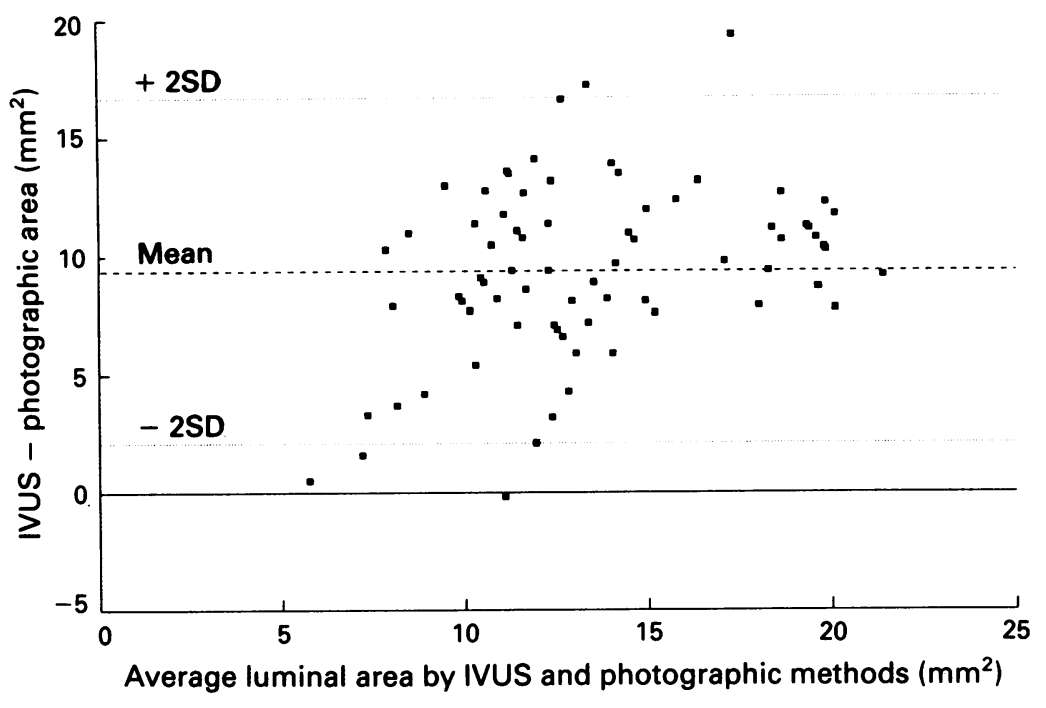

Figure 4 Difference between luminal area measured by the intravascular ultrasound system and that measured from photographs of vessel sections plotted against the mean of the two values. Fixed lines show the mean of the difference and $2 S D$ from this mean.

round by hand and measured by the Diasonics ultrasound machine. The image was orientated by placing a fine needle in front of the anterior wall of the artery before the first image. On completion of collection of the images the artery carriage was removed from the water bath. A fine line was drawn down the anterior wall of the artery with indelible ink and the upper end of the specimen was marked. The sutures were removed. The artery was then transferred to a shaped cutting block that supported the tissue while a multibladed cutter sliced it at levels that corresponded to the ultrasound images. The $3 \mathrm{~mm}$ segments that corresponded to each ultrasound image were photographed with the cut surface corresponding to the ultrasound image uppermost, with a small plastic block of known size for calibration. The area of the lumen was measured from the photographs with a digitising tablet and Apple Macintosh SE computer. Each specimen was then embedded in paraffin wax and his-

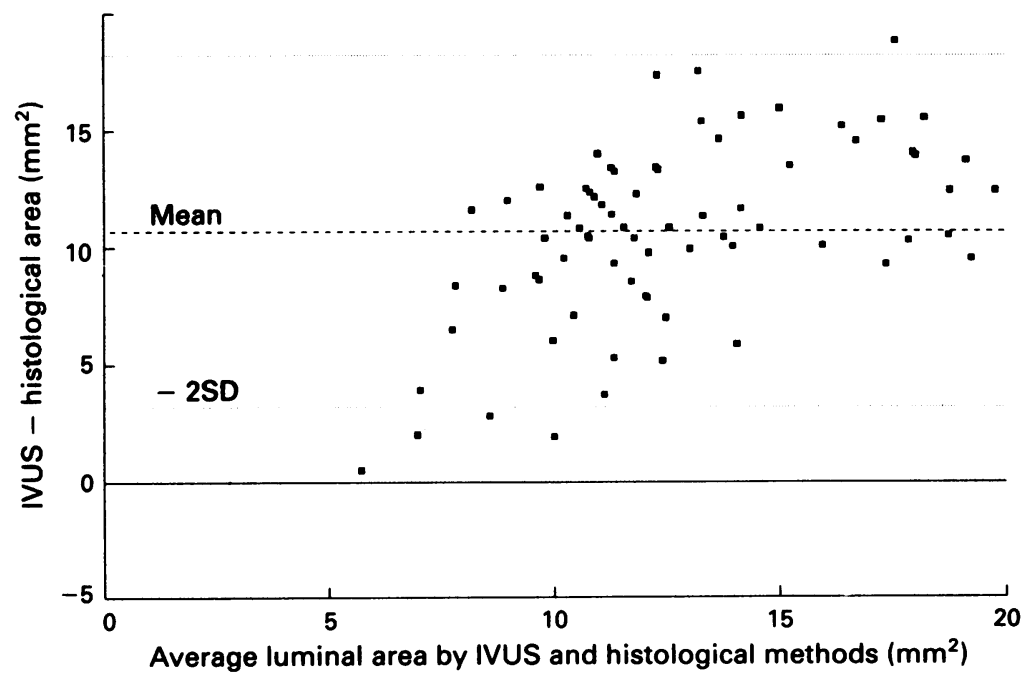

Figure 5 Difference between luminal area measured by the intravascular ultrasound system and that measured by microscopy of vessel sections plotted against the mean of the two values. Fixed lines show the mean of the difference and $2 S D$ from this mean. tological sections were made that corresponded to the ultrasound images. The histological slides were examined through an AMS quantifying microscope with video planimetry to measure the luminal area. On inspection the section was divided into four quadrants-from $45^{\circ}$ to $135^{\circ}, 135^{\circ}$ to $225^{\circ}, 225^{\circ}$ to $315^{\circ}$, and from $315^{\circ}$ to $45^{\circ}$. The presence or absence of atheroma and calcification was recorded for each segment by an independent observer. With the images recorded on videotape a similar procedure by another investigator was repeated for the ultrasound images by comparing each quadrant with adjacent quadrants and also adjacent segments. The luminal areas and qualitative judgements on the presence or absence of atheroma in the ultrasound, photographic, and histological images were compared.

For calibration two sections of polyethylene tube of known size (4.1 and $5.4 \mathrm{~mm}$ internal diameter) were placed on the artery carriage and their lumens were imaged and measured. The measurement was repeated five times for each tube.

\section{STATISTICAL ANALYSIS}

The measurements of luminal area taken from each method were compared with the method of limits of agreement ${ }^{5}$ to assess measurement bias and confidence intervals. The presence or absence of atheroma on histological examination was used to assess the sensitivity and specificity of the observations made from the ultrasound images.

A probability value of $p<0.05$ was considered statistically significant and confidence intervals were calculated as mean (2 SD) with 75 degrees of freedom.

\section{Results}

Figure 4 shows the results of a comparison of area of the vessel lumen measured by ultrasound and by photography of the vessel sections. Values of the area measured by ultrasound were on average $9.39 \mathrm{~mm}^{2}$ larger than the same area measured from photographs of the vessel slices (confidence intervals (CI) for an individual ultrasound area measurement was $2 \cdot 10-16 \cdot 7 \mathrm{~mm}^{2}>$ photographic area, CI for the mean difference was $8 \cdot 6-10 \cdot 2 \mathrm{~mm}^{2}$ ). A similar pattern was noted when measurements of area by ultrasound were compared with those made by the AMS quantifying microscope (fig 5). The ultrasound area was on average $10.7 \mathrm{~mm}^{2}$ greater than that measured histologically (CI for an individual ultrasound area $3 \cdot 2-18 \cdot 2 \mathrm{~mm}^{2}>$ histological area, CI for the mean difference $9.8-11.6 \mathrm{~mm}^{2}$ ). By contrast (fig 6) there was close agreement between the histological and photographic techniques (mean difference $-1.3 \mathrm{~mm}^{2}$, CI of an individual histological area measurement -4.3 to $+1.6 \mathrm{~mm}^{2}$ compared with the photographic area, CI for the mean difference -1.6 to $-0.97 \mathrm{~mm}^{2}$ ). The overestimation of area of the lumen by the intravascular ultrasound system in relation to the other two techniques was statistically and clinically significant. The wide 


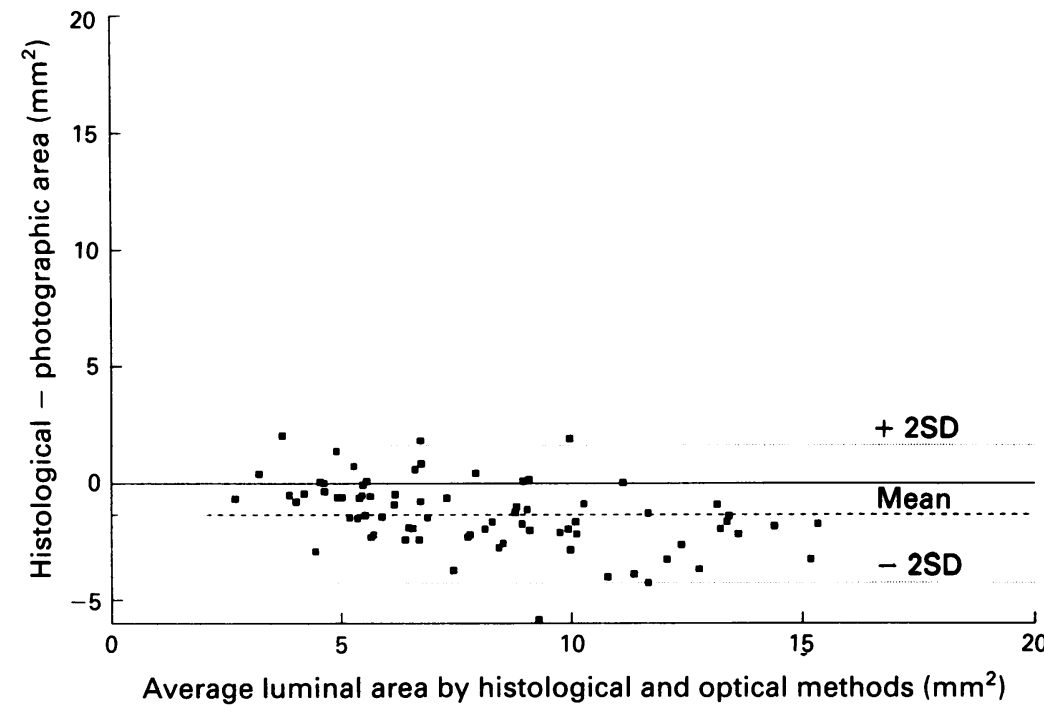

Figure 6 Difference between luminal area measured by microscopy of vessel sections and that measured from photographs (optical) of the sections plotted against the mean of the two values.

CI for luminal area measurements made with the ultrasound system also reduces its value. Interestingly the intravascular ultrasound system showed a much reduced bias when measuring the lumen of polyethylene tubes of known diameter (fig 7). Areas measured by ultrasound were only $2.14 \mathrm{~mm}^{2}$ greater than the actual area calculated from the mean of the two diameters measured by micrometer (CI for an individual measurement $0.22-4.06 \mathrm{~mm}^{2}, \mathrm{CI}$ for the mean difference $1.53-2.75 \mathrm{~mm}^{2}$ ). Eccentric positioning of the ultrasound imaging catheter within the polyethylene tube had little effect on the measured values.

The presence or absence of atheroma detected by ultrasound and histological examinations was compared. Overall $36 \%$ of the 304 quadrants studies had identifiable atheroma on histological examination. Ultrasound sensitivity was 0.593 and the specificity was 0.839 . The positive predictive value was 0.674 , and the relative risk $3 \cdot 139$.

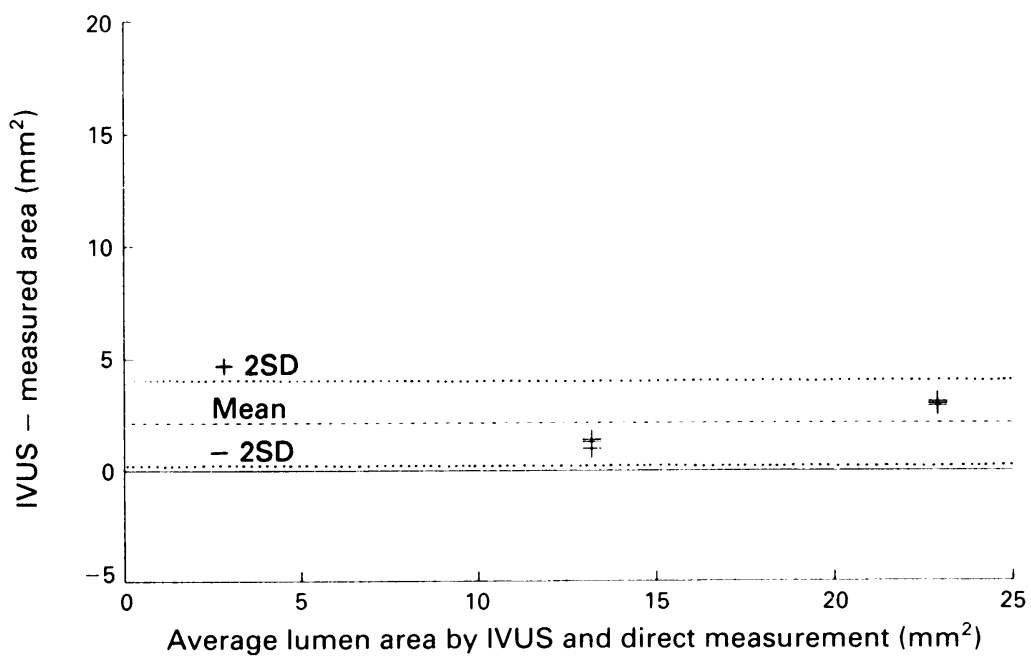

Figure 7 Difference between luminal area measured by the intravascular ultrasound system and the measured diameter of polyethylene tubes plotted against the mean of the two values. Fixed lines show the mean of the difference and $2 S D$ from this mean.

\section{Discussion}

The development of transcoronary treatments such as angioplasty, atherectomy, rotary ablation, and stent placement has led to a growth of interest in the internal morphology of the coronary arteries. The precise morphology of atheroma and its constitution may be important factors in determining the initial success rate of stenosis after these procedures. ${ }^{6}$ Interpretation of coronary angiography is subjective and a poor correlation between angiographic and post mortem pathological findings has been shown. ${ }^{78}$ Interest has centred on whether intravascular ultrasound can offer more accurate information on size of the lumen and atheroma morphology. Some studies have provided conflicting answers to this question.

With a $25 \mathrm{MHz}$ catheter Potkin and colleagues ${ }^{9}$ studied fixed and unfixed specimens of coronary artery and compared the luminal areas with those measured after histological fixation. They found a correlation coefficient $r=0.85$ (luminal area by ultrasound $=1.13 \times$ lumenal area by histology -0.58 ). Mean luminal area was $2.65 \mathrm{~mm}^{2}$. Gussenhoven et $a l^{10}$ used a $40 \mathrm{MHz}$ catheter to study peripheral arteries before histological fixation and sectioning. Ultrasound measurements correlated closely with histological measurements $(r=0.847)$ but the gradient of the relation diverged considerably from unity (ultrasound $=0.587 \times$ histology +11.8 ). Mean luminal area was $25 \mathrm{~mm}^{2}$.

Similarly variable results have been reported for the comparison of ultrasound luminal areas with those derived from angiography. Davidson $e t a l^{11}$ reported a high degree of correlation $(r=0.95)$ between luminal areas derived from ultrasound and digital subtraction angiograms in peripheral vessels, and another study reported good results $(r=0.80)$ in the coronary vessels although the luminal areas measured by ultrasound were on average $35 \%$ larger than those derived by angiography. ${ }^{12}$ Tobis $e t$ al ${ }^{13}$ reported a correlation coefficient of only 0.26 between ultrasound and angiographic measurements of the lumen in areas of vessels apparently normal by angiography.

The results of our study show a poor level of agreement between ultrasound measurements and cross sectional areas measured from scaled photographs and histological specimens. By contrast the photographic and histological measurements show close agreement. The measurements made by the photographic technique were individually calibrated reducing the risk of significant error. The only processing performed between ultrasound imaging and photographic measurements was sectioning of the specimens. Distortion of the specimens after sectioning was not seen reflecting the firm texture of the vessel after pressure fixation. The small difference between the areas measured from photographs and those taken from histological sections is probably due to shrinkage occurring during histological processing of the specimens.

The intravascular ultrasound system was calibrated with polyethylene tubing of known internal diameter. Ultrasound luminal area 
Figure 8 Typical intravascular ultrasound image. The dark central shadow $(A)$ is surrounded by a bright pericatheter halo. More distant vessel wall structure is clearly seen (C) but the exact boundary between lumen and intima is impossible to identify $(B)$.

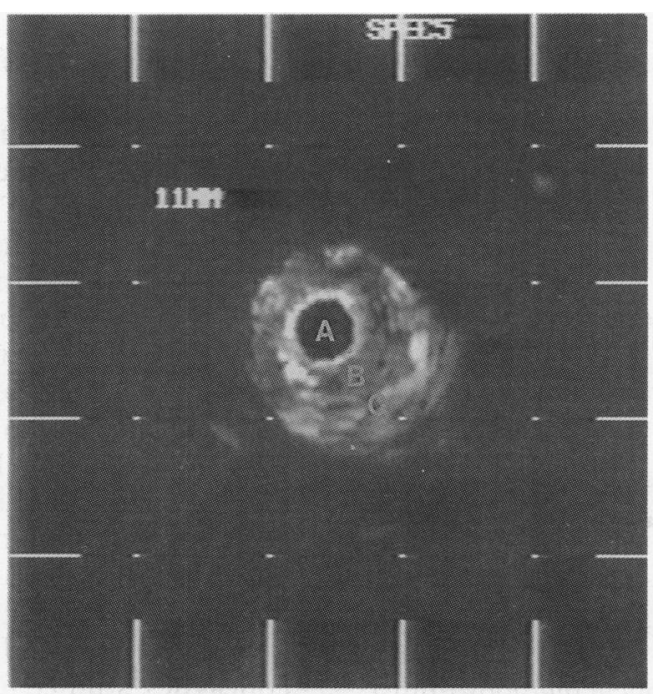

only slightly overestimated measured area with a difference in mean (CI) of 2.14 (1.53$2 \cdot 75) \mathrm{mm}^{2}$. Axial tilting of the catheter within the artery specimen could account for a small overestimate of area. The length $(2 \mathrm{~cm})$ of our arterial specimens, however, and the close support provided for the imaging catheter by the imaging jig make this explanation unlikely. Another possible explanation for the discrepancy in measurements of area of the lumen is the use of saline rather than blood in the immersion bath. Moriuchi et al $^{14}$ have considered this question in detail. They showed a small $(6 \%)$ increase in cross sectional area of measurements performed in saline rather than blood. Whereas this would not account for the large discrepancy in our measurements of ultrasound area made in coronary vessels it might account for the overestimation (roughly $10 \%$ ) of the area of the polyethylene tubes by the intravascular ultrasound system. Other groups have also shown that the use of saline in the specimen bath is unlikely to be a major source of error in measurement of luminal area. $^{15}$

There appears to be a fundamental difference in the error in measurement of luminal area when the intravascular ultrasound system is used in coronary arteries compared with polyethylene tubes. During interpretation of the coronary artery ultrasound images great difficulty was often encountered in finding the precise location of the boundary between the lumen and the intima, and the boundary was sometimes obscured by the near field ring shadows originating from the catheter itself (fig 8). It is likely that poor definition of this boundary and hence overestimation of the area of the vessel lumen explains the large bias noted. In the polyethylene tubes a very sharp image of the boundary between lumen and tubing was produced with more accurate measurement of luminal area.

Our original intention was to grade the appearance of the ultrasound images as previously defined. ${ }^{4}$ On the basis of peripheral vascular studies bright echoes with shadows behind indicate calcium deposits, bright echoes alone indicate collagen rich fibrous tissue, soft echoes indicate fibromuscular tissue, intimal proliferation, and diffuse lipid, whereas hypoechoic areas indicate significant pooling of lipid. Unfortunately the quality of the images that we obtained was not consistently good enough to make a classification. In view of this difficulty we compared each ultrasound and histological image quadrant by quadrant for the presence or absence of atheroma (each scored by an independent observer). Atheroma was defined on the ultrasound images as thickening of the vessel wall associated with one or more of the appearances defined above. Histological definition of atheroma excluded simple intimal thickening. Histological examination showed atheroma in $36 \%$ of the 304 quadrants examined.

Not surprisingly in view of the difficulty with identification of the luminal border and substructure of the vessel wall, sensitivity of the ultrasound technique for atheroma was low $(0.593)$, although with somewhat better specificity $(0 \cdot 839)$.

Despite numerous reports indicating that intravascular ultrasound is a more sensitive detector of atheroma within the coronary arteries than angiography, ${ }^{16}$ there have been no formal studies comparing its sensitivity with histological examination of the vessel. Those studies that have shown excellent correlation have used peripheral arteries with larger lumens and thicker walls. ${ }^{413}$ These peripheral vessels tend to produce superior ultrasound images.

POSSIBLE EXPLANATIONS OF THE DISCREPANCY BETWEEN THE ULTRASOUND AND HISTOLOGICAL DATA

The primary factor limiting the accuracy of measurement of luminal area and identification of atheroma in this study seems to be the quality of the ultrasound image and failure to identify the interface betwen lumen and intima. When a clear interface occurred as in the polyethylene tubes the system appears to measure the luminal area accurately. Several factors could be responsible for the reduced quality of image.

Single transducer catheters such as the one in this study use a rotating drive shaft to turn the transducer itself (as in this catheter) or an ultrasound mirror to reflect the signal from a fixed transducer. Both types of catheter are subject to non-uniform rotational distortion due to the limited torsional rigidity of the drive shaft. ${ }^{18}$ In other words the tip of the drive shaft may lag behind the motor drive unit by angles of up to $40^{\circ}$ depending on angle of the catheter and speed of rotation. This lag may vary during the course of each rotation due to the "slip and stick" phenomenon and this in turn can produce apparent compression or expansion of segments of the artery wall. The $4.8 \mathrm{~F}$ catheter in this study is one of the smallest catheters used for such imaging studies and may be more prone to lack of torsional rigidity in the drive shaft for this reason. Such problems could account for some of the variability in measurements of the luminal area and discrepancies in the localisation of atheroma.

One feature of the images produced by this catheter was the pericatheter near field effect 
that often obscured the exact junction between the lumen and the intima when the catheter was positioned close to the vessel wall (fig 8 ). This effect may be less troublesome in catheters with a rotating mirror rather than a rotating transducer. In this type of catheter the near field of the transducer falls partly or wholly within the distance between the transducer and the mirror and therefore is not apparent on the processed image of the vessel wall. ${ }^{19}$ The $4.8 \mathrm{~F}$ catheter used in this study must have its tip filled with sterile water before use and the inclusion of air bubbles at this stage can cause degradation of the image. The catheter tip was, however, carefully filled in accordance with the manufacturer's instructions and there was no evidence of bubble artefact on the images produced.

The small size of this catheter may contribute to the difficulty of getting a clear image. As catheter size is reduced, the aperture of the transducer element is also reduced accentuating lateral resolution artefacts. ${ }^{20}$ This may produce a smearing of the image particularly at interfaces between atheromatous plaques and the normal arterial wall.

It is important to consider whether the formalin pressure fixation technique used for the arterial specimens could alter the quality of the ultrasound images and thus account for some of the difficulties we found. It is known that formalin fixation can increase the strength of the reflected ultrasound signal. ${ }^{21}$ Tobis $e t$ al $^{22}$ however, showed only a small change in luminal area measured by ultrasound after formalin fixation and made no comment concerning any change in the appearance of the vessel. Also the pressure fixation technique may have some benefits. There has been much discussion about the three layered appearance of intravascular ultrasound images of muscular arteries that has been noted by some authors ${ }^{43}$ but not by others. ${ }^{24} 25$ Such differences may reflect differences in preparation of the specimens. The appearance of an inner ring on intravascular ultrasound images is exaggerated in nonpressure fixed specimens. This may be due to contraction and corrugation of the internal elastic lamina. ${ }^{26}$ The technique of pressure fixation that we have used is, we think, most likely to retain the morphology and qualitative ultrasound features of the artery specimens when compared with in vivo arteries.

Although $20 \mathrm{MHz}$ single transducer probes can produce excellent images in the peripheral vessels the results of our study would suggest that further improvements in catheter design are required to get clinically useful images of the coronary arteries. A number of approaches may improve the quality of the images from such catheters. A higher ultrasound frequency enables improved resolution albeit with some loss of penetration. Increasingly, images systems for coronary imaging catheters use ultrasound frequencies in the $30-50 \mathrm{MHz}$ range. The use of an array of ultrasound crystals in place of a single crystal offers an alternative means of improving image quality. Computer processing of the image may enable the effective focal length of such a transducer to be varied.

In conclusion the intravascular ultrasound system evaluated in our study lacks the ability to produce accurate information on the dimensions and morphology of medium sized coronary arteries although the system is reported to produce excellent images of larger peripheral arteries. Further technical developents are required before this system can produce useful images of the coronary arteries in vivo.

We are grateful to Diasonics UK for the loan of the ultrasound We are grateful to Diasonics Physics department at St George's Hospital for their assistance with construction of the specimen jig and cutter.

1 Bom N, ten Hoff H, Lancée CT, Gussenhoven WJ, Bosch JG. Early and recent intraluminal ultrasound devices. Int $J$ Card Imaging 1989;4:79-88.

2 Nissen SE, Grines CL, Gurley JC, et al. Application of a new phased-array ultrasound imaging catheter in the assessment of vascular dimensions. Circulation 1990;81:660-6.

3 Panadian NG, Kreis A, O'Donnell T. Intravascular ultrasound estimation of arterial stenosis. J Am Soc Echocardiogr 1989;2:390-7.

4 Gussenhoven WJ, Essed CE, Frietman P, et al. Intravascular echographic assessment of vessel wall characteristics: a correlation with histology. Int J Card Imaging 1989;4:105-16.

5 Bland JM, Altman DG. Statistical methods for assessing agreement between two methods of clinical measurement. Lancet 1986;i:307-10.

6 Liu MW, Roubin GS, King SB. Restenosis after coronary angioplasty. Circulation 1989;79:1374-87.

7 De Rouen T, Murray JA, Owen W. Variability in the analysis of coronary arteriograms. Circulation 1977; 55:324-8.

8 Grondin CM, Dyrda I, Pasternac A, Campeau L, Bourassa MG, Lesperance J. Discrepancies between cineangioMG, Lesperance $J$. Discrepancies between cineangiographic and postmortem findings in patients with coronary
artery disease and recent myocardial revascularisation. artery disease and recent
Circulation 1974;49:703-8.

9 Potkin BN, Bartorelli AL, Gessert JM, et al. Coronary artery imaging with intravascular high-frequency ultrasound. Circulation 1990;81:1575-85.

10 Gussenhoven EJ, Essed CE, Lancée CT, et al. Arterial wall characteristics determined by intravascular ultrasound imaging: an in vitro study. $J$ Am Coll Cardiol 1989;14: 947-52.

11 Davidson CJ, Sheikh KH, Harrison K, et al. Intravascular ultrasound versus digital subtraction angiography: a human in vivo comparison of vessel size and morphology [abstract]. J Am Coll Cardiol 1990;15:16.

12 Nissen SE, Gurley JC, Booth DC. In vivo assessment of human coronary minimum luminal diameter with a multielement intravascular ultrasound catheter: comparison to quantitative cineangiography [abstract]. J Am Coll quantitative cinean
Cardiol 1990;15:29.

13 Tobis JM, Mallery J, Mahon D, et al. Intravascular ultrasound imaging of human coronary arteries in vivo. ultrasound imaging of hum

14 Moriuchi M, Tobis JM, Mahon D, et al. The reproducibility of intravascular ultrasound imaging in vitro. $\mathrm{J} \mathrm{Am} \mathrm{Soc}$ Echocardiogr 1990;3:444-50.

15 Hodgson J McB, Graham SP, Savakus AD, et al. Clinical percutaneous imaging of coronary anatomy using an overthe-wire ultrasound catheter system. Int $J$ Cardiac Imaging 1989;4:187-93.

16 Keren G, Douek P, Hansch E, Milner MR, Pichard AD Leon MB. Intravascular ultrasound characteristics of atheroma in angiographically "normal" segments [abstract]. J Am Coll Cardiol 1991;17:217.

17 Tobis JM, Mahon D, Lehmann K, McRae M, Henry WL. The sensitivity of ultrasound imaging compared with angiography for diagnosing coronary atherosclerosis angiography for diagnosing coro

18 ten Hoff H, Korbijn A, Smit TH, Klinkhammer JFF, Bom $\mathrm{N}$. Imaging artefacts in mechanically driven ultrasound N. Imaging artefacts in mechanically driven
catheters. Int J Card Imaging 1989;4:195-9.

19 Mallery JA, Tobis JM, Griffith J, et al. Assessment of normal and atherosclerotic arterial wall thickness with an and atherosclerotic arterial wall thickness with an
intravascular ultrasound imaging catheter. Am Heart $J$ intravascular ultraso

20 Crowley RJ, von Behren PL, Couvillon LA, Mai DE, Abele JE. Optimized ultrasound imaging catheters for use in the vascular system. Int J Card Imaging 1989;4:145-51.

21 Gussenhoven EJ, Essed CE, Lanceé CT, et al. Arterial wall characteristics determined by ultrasound imaging: an in vitro study. J Am Coll Cardiol 1989;14:947-52.

22 Tobis JM, Mallery JA, Gessert J, et al. Intravascular ultrasound cross-sectional imaging before and after balloon angioplasty in vitro. Circulation 1989;80:873-82.

23 Pandian NG, Kreis A, Weintraub A, et al. Real-time intravascular ultrasound imaging in humans. $A m$ Cardiol 1990;65:1392-6.

24 Gurley JC, Nissen SE, Diaz C, Fischer C, O'Connor WN, DeMaria AN. Is the tri-layer arterial appearance an artefact? Differences between in vivo and in vitro intravascular ultrasound [abstract]. J Am Coll Cardiol 1991;17:112.

25 Fitzgerald PJ, St Goar FG, Kao AK, et al. Intravascular ultrasound imaging of coronary arteries: is three layers the norm? [abstract]. J Am Coll Cardiol 1991;17:217. 26 Webb JG, Yock PG, Slepian MJ, et al. Intravascular ultrasound: significance of the three-layered appearance of normal muscu 